九州大学学術情報リポジトリ

Kyushu University Institutional Repository

\title{
Expression of activated signal transducer and activator Stat3 as a predictor of poor prognosis in cervical cancer
}

Sonoda, Kenzo

Department of Obstetrics and Gynecology, Graduate School of Medical Sciences, Kyushu University

http://hdl. handle. net/2324/26436

出版情報：Expert Review of Obstetrics and Gynecology. 5 (2)，pp.161-164，2010-03. Expert Reviews

バージョン :

権利関係 : (C) 2010 Expert Reviews Ltd 
Expression of activated signal transducer and activator of transcription-3 (Stat3) as a predictor of poor prognosis in cervical cancer

Running title: Stat3 expression in cervical cancer

Evaluation of: Takemoto S, Ushijima K, Kawano K et al. Expression of activated signal transducer and activator of transcription-3 predicts poor prognosis in cervical squamous-cell carcinoma. Br. J. Cancer 101, 967-972 (2009).

Kenzo Sonoda, MD, PhD

Department of Obstetrics and Gynecology, Graduate School of Medical Sciences, Kyushu University, Maidashi 3-1-1, Higashi-ku, Fukuoka 812-8582, Japan.

Tel: 81-92-642-5395 Fax: 81-92-642-5414

E-mail: kenzo@med.kyushu-u.ac.jp

Keywords: cervical cancer; Stat3; VEGF; Bcl-xL; molecular target therapy 


\section{Abstract}

Stat3 is activated through phosphorylation (p-Stat3) whereupon it enhances the transcription of several molecules involved in cell survival and proliferation. The constitutive activation of Stat 3 has been reported in various types of malignancies, and its expression has been shown to indicate a poor prognosis. However, the clinical significance of Stat3 expression in cervical cancer has seldom been evaluated. Using immunohistochemical methods, Takemoto et al. investigated the association between p-Stat3 expression and clinicopathological variables. The expression of p-Stat3 was significantly correlated with stage, tumor size, lymph vascular space invasion, and lymph node metastasis. Furthermore, p-Stat3 expression was statistically indicative of a poor prognosis. These data demonstrate the clinical significance of p-Stat3 expression. Continued studies of Stat3 function may help in the development of novel therapeutic strategies for cervical cancer. 


\section{Introduction}

Cancer research is now focusing on the factors that increase the aggressive behavior of human malignancies, including cervical cancer [1]. Cervical cancer is the second most common cancer among women worldwide and the seventh overall [2]. In general terms, it is much more common in developing countries, with an estimated 493,000 new cases and 274,000 deaths in 2002. Tumor volume, histologic type, stage, lymph vascular space invasion, and lymph node metastasis have been reported as prognostic factors in surgically treated cervical cancer [3]. Over the past decade, women with cervical cancer of all stages have benefited from tremendous improvements in the treatment of this disease. Treatment of invasive cancer involves appropriate management for both the primary lesion and potential sites of metastatic disease. Both surgery and radiation therapy may be used for primary treatment, although chemotherapy for advanced or recurrent disease has been and continues to be considered palliative [4]. On the other hand, novel anti-cancer therapeutic strategies have been developed by targeting molecules which are involved in cell survival and proliferation, approaches based upon advances in our understanding of the molecular basis of cancer [5-7].

Stat3 was first identified as a DNA-binding factor that selectively binds to the interleukin (IL)-6 responsive element in the promoter of 
acute-phase genes from IL-6-stimulated hepatocytes [8]. The gene that encodes Stat3 is located on chromosome $17 \mathrm{q} 21$, and it encodes a $92 \mathrm{kDa}$ protein. Stat 3 is activated by many cytokines and growth factors, including epidermal growth factor (EGF), platelet-derived growth factor, and IL-6 as well as by oncogenic proteins, such as Src and Ras [9]. Stat3 normally resides in the cytoplasm and can be activated through phosphorylation at tyrosine residue 705. Cytoplasmic p-Stat3 dimerizes and translocates to the nucleus, where its occupation of specific DNA-binding sites results in the increased transcription of molecules that are involved directly in development, differentiation, proliferation, motility, and survival. In fact, Stat3 deletion in mice leads to embryonic lethality [10]. Furthermore, conditional knockout mice have shown the pleiotropic roles of Stat3 in many organs and cell types, including the heart, skin, lymphocytes, monocytes, neutrophils, mammary epithelium, liver, and neurons [11]. For example, cardiomyocyte-specific conditional Stat3 knockout mice showed enhanced susceptibility to cardiac injury caused by myocardial ischemia, systemic inflammation, or drug toxicity. These mice were also more prone to the pathogenesis of age-related heart failure.

Perhaps due in part to the numerous roles of Stat3 in a wide variety of physiologic processes, Stat3 activation in normal cells is transient and tightly controlled. In contrast, constitutively activated Stat 3 protein has 
been found in various types of tumors, including leukemia and cancers of the breast, head and neck, melanoma, prostate, and pancreas [9]. Stat3 has been classified as a proto-oncogene because an activated form of Stat3 can mediate oncogenic transformation in cultured cells and tumor formation in nude mice. For example, activation of Stat3 is required for cell transformation by oncogenic Src [12]. In addition, overexpression of constitutively activated Stat3 in immortalized NIH-3T3 fibroblast cells [13] and immortalized human mammary epithelial cells induces cellular transformation and tumor formation in nude mice [14]. Constitutive Stat3 signaling participates in oncogenesis by stimulating cell proliferation, promoting angiogenesis, mediating immune evasion, and conferring resistance to apoptosis induced by conventional therapies [15-17].

So far, only a few papers have analyzed Stat 3 in the context of gynecological malignancies. Rosen et al. analyzed p-Stat3 expression in ovarian cancer and reported that high p-Stat 3 expression in tumor tissue was associated with high levels of HER-2/neu, EGF receptor, and Ki-67 [18]. Though no correlation was observed between overall p-Stat3 levels and other clinicopathological variables, high nuclear expression of p-Stat3 was linked with poor overall survival. In cervical cancer, Chen et al. showed that $\mathrm{p}$-Stat 3 expression was associated with increased expression of downstream anti-apoptotic genes, Bcl-xL, survivin, and $M c l-1$. In spite of 
those intriguing findings, little is known about the prognostic relevance of p-Stat3 [19]. To clarify whether p-Stat3 is a valuable prognostic indicator in cervical cancer, Takemoto et al. [1] examined the following: (1) p-Stat3 expression in cervical cancer cells, (2) the relationship between p-Stat3 expression and several clinicopathological variables, and (3) the contribution of $\mathrm{p}$-Stat3 expression to prognosis.

\section{Methods and results}

Takemoto et al. investigated p-Stat3 expression in cervical cancer specimens through the use of immunohistochemical methods with anti-p-Stat3 antibody [1]. They obtained specimens from 125 patients with invasive squamous cell carcinoma who had undergone extended hysterectomy with pelvic lymphadenectomy. Specimens were considered positive when nuclear staining was observed. The authors analyzed the association between p-Stat 3 expression and clinicopathological variables. They also evaluated p-Stat 3 expression as a prognostic factor related to overall survival (OS) and disease-free survival (DFS) rates. In addition, multivariate analysis was conducted using the Cox proportional hazard method. The expression of p-Stat3 was reported to correlate with increased expression of vascular endothelial growth factor (VEGF), an angiogenic factor, and Bcl-xL, an anti-apoptotic molecule [16, 19]. Therefore, the 
expression of VEGF and Bcl-xL was measured by Western blot after Stat3-specific small interfering RNA (siRNA) was transfected into the cervical cancer cell line SKG II in order to suppress Stat3 expression.

Results showed no staining for p-Stat3 in normal cervical tissues. Of 125 tumor samples examined, 71 cases $(56.8 \%)$ were positive and 54 (43.2\%) were negative for $\mathrm{p}$-Stat3 expression. Expression of $\mathrm{p}$-Stat3 showed significant associations with advanced stage $(P=0.006)$, large tumor size $(P=0.001)$, lymph vascular space involvement $(P=0.004)$, and lymph node metastasis $(P=0.007)$. The positive ratio of $\mathrm{p}$-Stat3 expression in tumor tissues was significantly related to the number of lymph nodes involved in metastasis $(P=0.022)$. With regard to patient outcome, expression of p-Stat3 was inversely associated with OS and DFS rates $(P=$ 0.006 and $P=0.010$, respectively). According to multivariate analysis, deep stromal invasion was the only independent prognostic factor for DFS. Although p-Stat3 expression was not an independent prognostic factor for OS or DFS, the hazard ratio was the highest of the significant prognostic factors by univariate analysis (data not shown). Introduction of Stat3-specific siRNA into SKG II cells inhibited the expression of p-Stat3 as well as VEGF and Bcl-xL.

\section{Discussion and significance}


This report is the first to examine p-Stat3 expression as a prognostic factor in cervical cancer. Until now, the expression of p-Stat3 has been studied and recognized as a predictor of poor survival in several human malignancies [9]. Immunoexpression of p-Stat3 has also been linked with several clinicopathological parameters, including tumor diameter and lymph node metastasis. In this study, p-Stat3 staining was significantly associated with advanced stage, large tumor size, lymph vascular space involvement, and lymph node metastasis which are predictors of poor prognosis in cervical cancer [3]. Expression of p-Stat3 was not identified as an independent prognostic factor by multivariate analysis. The authors suggested possible reasons for this result, as p-Stat 3 expression correlated with other clinicopathological factors.

In a previous immunohistochemical study of p-Stat3 in cervical cancer [19], the frequency of p-Stat3 positivity was lower (24\% of 104 total cases) and no significant correlation was detected between p-Stat3 expression and any clinicopathological variable, including age, histology, grade, stage, or lymph node metastasis. Takemoto et al. discussed factors which might have contributed to the differences in results. First, with regard to histologic subtype, the previous study included adenocarcinoma and microinvasive carcinoma. Second, the definition of positive expression differed, as the previous study defined weak-staining specimens as negative. 
Finally, the previous report provided an insufficient description of clinicopathological variables. So far, only two papers concerning p-Stat3 expression in cervical cancer have been published. However, the accumulated data indicate that activated Stat3 may have clinical significance in determining the outcome of patients. Further investigation is necessary to understand the biological significance of Stat 3 which likely influences proliferation, and the angiogenic and metastatic potential of cancer cells.

\section{Expert commentary}

With advancements in our understanding of the basic mechanisms of oncogenesis, invasion, and metastasis, we have gained a greater appreciation for the critical factors that influence tumor cell biology. Some of these factors are involved in the development of resistance to conventional treatment. The important task now facing researchers and clinicians is to translate these discoveries into novel therapeutics that will improve patient outcome. Specifically, we must target new drugs against the molecules which are pivotal in cell proliferation, promotion of angiogenesis, mediation of immune evasion, and tumor metastasis [5, 6]. So far, the development of novel therapeutics has largely focused on inhibitors of signal transduction, particularly protein kinases [7]. Presently, 
the Food and Drug Administration in the United States has approved over ten protein kinase inhibitors and over 100 kinase-targeted agents are currently undergoing clinical evaluation.

On the other hand, recent advances in investigating the role of Stat3 in tumor progression indicate that Stat 3 signaling might be a common molecular target for blocking tumor spread. Several strategies have been pursued for developing inhibitors of Stat3, based on the complex nature of its gene expression and activity. Strategies to abolish Stat3 expression include the delivery of antisense oligonucleotides and siRNA, whereas approaches for inhibition of Stat3 activity include the use of tyrosine kinase inhibitors, phosphopeptides, G-quartet oligodeoxynucleotides, decoy oligonucleotides, and small-molecule compounds that inhibit DNA-binding [9]. The administration of Stat3 decoy oligonucleotides resulted in abrogation of Stat 3 activation and decreased tumor volumes by inducing apoptosis [20]. This effect was augmented when combined with cisplatin.

Among therapeutic strategies targeting Stat3, significant advances have been made using small molecule-based approaches, such as IS3 295, SD-1029, and WP1066, which have the most immediate therapeutic potential. IS3 295, a novel platinum compound, has also been identified in the National Cancer Institute (NCI) Diversity Set as a selective Stat3 inhibitor [21]. It interacts with Stat3 directly and inhibits Stat3 binding to 
specific DNA response elements. Unlike its prototype (cisplatin), IS3 295 has no effects on Akt and mitogen-activated protein kinase family pathways. IS3 295 inhibits the Stat3-regulated molecules cyclin D1, Bcl-xL, and VEGF. It also selectively attenuates Stat3 signaling, thereby inducing cell growth arrest and apoptosis. SD-1029 was also identified as a micromolar inhibitor of IL-6- or oncostatin-induced Stat3 nuclear translocation among the NCI Diversity set [22]. Biochemical analysis shows that SD-1029 inhibits tyrosyl phosphorylation of Stat3 implicating SD-1029 as an inhibitor of Janus-activated kinase (JAK). Further analysis shows that this compound inhibits tyrosyl phosphorylation of JAK-2 isoenzyme. The anti-apoptotic proteins $\mathrm{Bcl}-\mathrm{xL}$ and survivin, target proteins of activated Stat3, are down-regulated by SD-1029 resulting in the induction of apoptosis in several human breast and ovarian cancer cell lines. SD-1029 also enhances apoptosis induced by paclitaxel in ovarian cancer cells. WP1066, another JAK-2 inhibitor, suppresses the growth of malignant glioma cells both in vitro and in vivo [23]. It induces the expression of co-stimulatory molecules in peripheral macrophages and tumor-infiltrating microglia, stimulates the production of immune-stimulatory cytokines (IL-2, IL-4, IL-12, and IL-15), and induces proliferation of effector $\mathrm{T}$ cells from glioblastoma multiforme patients. Moreover, there have been several promising small substances reported: 
JSI-124 (an inhibitor of JAK-2), STA-21 (an inhibitor of Stat3 dimerization, DNA binding, and nuclear translocation), and the novel platinum compound CPA-7 [9]. A few Stat3-specific inhibitors, including OPB-31121, have entered into clinical trials [24]. At this point, based on the evidence from studies using relevant animal models, it is anticipated that Stat3 inhibitors should have only limited toxicity to normal cells because Stat3 activation in normal cells is transient and tightly controlled, and normal cells are not dependent on aberrant Stat3 activation for growth and survival $[11,25]$. Nonetheless, the toxicity and side effects of Stat3 inhibitors must be thoroughly investigated in animal models and ultimately in clinical trials, with careful assessment of both benefits and risks to patients.

Although the mechanism of constitutive activation of Stat3 has not been clarified in human malignancies, therapeutics targeting Stat3 activation might be an effective strategy. Definitive randomized clinical trials of Stat 3 inhibitors either alone or in combination with chemotherapy are required to ultimately determine their impact in cancer therapy.

\section{Five-year view}

Cervical cancer is a serious health problem, with nearly a half million women annually developing the disease worldwide. Although most 
women with early-stage tumors can be cured by improved therapeutics, treatment of advanced and recurrent cervical cancer remains largely ineffective. On the other hand, modern molecular and cellular biotechnological methods have provided new insights into this disease. Takemoto et al. showed the clinical significance of activated Stat3 expression as a predictor of patients' prognosis. The growing list of cases where suppression of Stat3 signaling leads to the demise of tumor cells establishes that these molecules contribute to the cancerous phenotype and provides hope that the near future will bring therapeutics targeting the activated Stat3 molecule.

\section{Key issues}

- Immunohistochemical analysis has shown that expression of p-Stat3 was associated with stage, tumor size, lymph vascular space involvement, and lymph node metastasis in cervical cancer. In addition, the frequency of p-Stat3 expression in tumor tissues was significantly related to the number of lymph node metastasis.

- Expression of p-Stat3 was inversely associated with OS and DFS rates of cervical cancer patients.

- Introduction of Stat3-specific siRNA into the cervical cancer cell line SKG II inhibited the expression of p-Stat3 as well as VEGF and 
Bcl-xL.

- Stat3 might be a promising target in anti-cancer molecular therapeutics.

However, better understanding of Stat3 activation in human malignancies is required. 


\section{References}

Papers of special note have been highlighted as:

* of interest

** of considerable interest

1. Takemoto S, Ushijima K, Kawano $\mathrm{K}$ et al. Expression of activated signal transducer and activator of transcription-3 predicts poor prognosis in cervical squamous-cell carcinoma. Br. J. Cancer 101, 967-972 (2009).

**This report is the first to show p-Stat3 expression acts as a predictor of poor prognosis of cervical cancer.

2. Parkin DM, Bray F, Ferlay J, Pisani P. Global cancer statistics, 2002. CA. Cancer J. Clin. 55, 74-108 (2005).

3. Randall ME, Michael H, Long H 3rd, Tedjarati S. Uterine cervix. In: Principles and Practice of Gynecologic Oncology. $5^{\text {th }}$ ed. Barakat RR, Markman M, Randall ME (Eds.), Lippincott Williams \& Wilkins, Philadelphia, 623-682 (2009).

4. Waggoner SE. Cervical cancer. Lancet 361, 2217-2225 (2003).

5. Schwartz GK, Shah MA. Targeting the cell cycle: A new approach to cancer therapy. J. Clin. Oncol. 23, 9408-9421 (2005).

6. Van Waes C. Nuclear factor- $\kappa \mathrm{B}$ in development, prevention, and therapy of cancer. Clin. Cancer Res. 13, 1076-1082 (2007). 
7. Roberts PJ, Der CJ. Targeting the Raf-MEK-ERK mitogen-activated protein kinase cascade for the treatment of cancer. Oncogene 26, 3291-3310 (2007).

8. Wegenka UM, Buschmann J, Lutticken C, Heinrich PC, Horn F. Acute-phase response factor, a nuclear factor binding to acute-phase response elements, is rapidly activated by interleukin-6 at the posttranslational level. Mol. Cell Biol. 13, 2762-2788 (1993).

9. Huang S. Regulation of metastases by signal transducer and activator of transcription 3 signaling pathway: Clinical implications. Clin. Cancer Res. 13, 1362-1366 (2007).

**This paper explains both the biological and clinical significance of Stat3.

10. Shen $\mathrm{Y}$, Schlessinger K, Zhu X et al. Essential role of STAT3 in postnatal survival and growth revealed by mice lacking STAT3 serine 727 phosphorylation. Mol. Cell Biol. 24, 407-419 (2004).

11. Levy DC, Lee CK. What does Stat3 do? J. Clin. Invest. 109, 1123-1148 (2002).

12. Yu CL, Meyer DJ, Campbell GS et al. Enhanced DNA-binding activity of a Stat3-related protein in cells transformed by the Src oncoprotein. Science 269, 81-83 (1995). 
13. Ram PT, Horvath CM, Iyengar R. Stat3-mediated transformation of NIH-3T3 cells by the constitutively active Q205L Galphao protein. Science 287, 142-144 (2000).

14. Dechow TN, Pedranzini L, Leitch A et al. Requirement of matrixmetalloproteinase-9 for the transformation of human mammary epithelial cells by Stat3-C. Proc. Natl. Acad. Sci. USA. 101, 10602-10607 (2004).

15. Real P, Sierra A, De Juan A, Segovia J, Lopez-Vega J, Fernandez-Luna J. Resistance to chemotherapy via Stat3-dependent overexpression of Bcl-2 in metastatic breast cancer cells. Oncogene 21, 7611-7618 (2002).

16. Wei L, Kuo M, Chen $\mathrm{C}$ et al. Interleukin-6 promotes cervical tumor growth by VEGF-dependent angiogenesis via a STAT3 pathway. Oncogene 22: 1517-1527 (2003).

17. Wang T, Niu G, Kortylewski $M$ et al. Regulation of the innate and adaptive immune responses by Stat-3 signaling in tumor cells. Nat. Med. $10,48-54$ (2004).

18. Rosen DG, Mercado-Uribe I, Yang G et al. The role of constitutively active signal transducer and activator of transcription 3 in ovarian tumorigenesis and prognosis. Cancer 107, 2730-2740 (2006). 
*This report is the first to show high p-Stat3 expression exists in ovarian cancer and associates with poor patient survival.

19. Chen CL, Hsieh FC, Lieblein JC et al. Stat3 activation in human endometrial and cervical cancers. Br. J. Cancer 96, 591-599 (2007). *This report is the first to show Stat3 is activated in endometrial and cervical cancer.

20. Xi S, Gooding WE, Grandis JR. In vivo antitumor efficacy of STAT3 blockade using a transcription factor decoy approach: implications for cancer therapy. Oncogene 24, 970-979 (2005).

21. Turkson J, Zhang S, Mora LB, Burns A, Sebti S, Jove R. A novel platinum compound inhibits constitutive Stat 3 signaling and induces cell cycle arrest and apoptosis of malignant cells. J. Biol. Chem. 280, 32979-32988 (2005).

22. Duan Z, Bradner JE, Greenberg E et al. SD-1029 inhibits signal transducer and activator of transcription 3 nuclear translocation. Clin. Cancer Res. 12, 6844-6852 (2006).

23. Hussain SF, Kong LY, Jordan J et al. A novel small molecule inhibitor of signal transducers and activators of transcription 3 reverses immune tolerance in malignant glioma patients. Cancer Res. 67, 9630-9636 (2007). 
24. United States National Institute of Health (http://www.nih.gov), last accessed on December/28/2009. Clinical trials.

25. Yu H, Jove R. The stats of cancer-New molecular targets come of age. Nat. Rev. Cancer 4, 97-105 (2004).

**This manuscript describes Stat3 as a promising therapeutic target. 